\title{
A INFLUÊNCIA DA ANSIEDADE NO DISTÚRBIO DA PARALISIA DO SONO SOB O OLHAR DA TERAPIA COGNITIVO COMPORTAMENTAL
}

\author{
THE INFLUENCE OF ANXIETY IN THE DISORDER OF SLEEP PARALYSIS UNDER \\ THE VIEW OF BEHAVIORAL COGNITIVE THERAPY
}

\author{
Lílian Neves Ribeiro da Costa ${ }^{1}$
}

RESUMO: Está presente pesquisa se propõe uma análise sobre a relação entre o transtorno de ansiedade e o distúrbio da paralisia do sono trazendo uma perspectiva da terapia cognitivo comportamental para contribuir com um enriquecimento a luz de uma abordagem pioneira em assuntos cognitivos comportamentais que está a todo momento contribuindo com estudos e explicações a respeito das subjetividades dos indivíduos. O objetivo geral deste trabalho se constituiu na investigação de como o transtorno de ansiedade pode estar intimamente ligado ao distúrbio da paralisia do sono trazendo a perspectiva da terapia cognitivo comportamental. Chegou-se à conclusão de que é correto afirmar que assim como a ansiedade é um potencial influenciador para o surgimento do distúrbio da paralisia do sono, o próprio distúrbio em si também pode vir a ser um possível gerador ou potencial influenciador para o transtorno de ansiedade. Sendo assim, se fez possível concluir que um é potencializador e possível influenciador do outro, e de certa forma, ambos se complementam. A metodologia que se utilizou esta pesquisa foi de caráter qualitativo, estando presente também na abordagem metodológica a pesquisa bibliográfica. Esta pesquisa teve ainda como contribuição social o auxílio informativo acerca da paralisia do sono e também a cerda se sua ligação com o transtorno de ansiedade, visto que essa temática não é devidamente explorada, investigada e estudada. A contribuição científica também foi de grande valia uma vez que a escassez de assuntos a respeito da paralisia do sono é grande no meio dos estudos científicos a respeito do tema.

Palavras- Chave: Ansiedade. Paralisia do sono. Disturbio. Paralisia Cognitivo Comportamental.

ABSTRACT: This research proposes an analysis of the relationship between anxiety disorder and sleep paralysis disorder, bringing a perspective of cognitive behavioral therapy to contribute to an enrichment in light of a pioneering approach to cognitive behavioral issues that is in full swing. moment contributing studies and explanations about the subjectivities of individuals. The general objective of this work was to investigate how anxiety disorder can be closely linked to sleep paralysis disorder, bringing the perspective of cognitive behavioral therapy. It was concluded that it is correct to say that just as anxiety is a potential influencer for the emergence of sleep paralysis disorder, the disorder itself can also become a possible generator or potential influencer for anxiety disorder. . Thus, it was possible to conclude that one is a potentializer and a possible influencer of the other, and in a way, both complement each other. The methodology used in this research was qualitative,

${ }^{1}$ Graduanda do $9^{\circ}$ período da graduação em psicologia pela Faculdade de ciências humanas de Olinda (FACHO).E-mail: 1ilianrcostaoz@gmail.com 
with bibliographic research also present in the methodological approach. This research also had as a social contribution the informative help about sleep paralysis and also the bristle if its connection with the anxiety disorder, since this theme is not properly explored, investigated and studied. The scientific contribution was also of great value since the scarcity of subjects regarding sleep paralysis is great in the midst of scientific studies on the subject.

Keywords: Anxiety. Sleep paralysis. Disturb. Cognitive Behavioral Paralysis.

\section{INTRODUÇÃO}

O presente trabalho se baseia no estudo a respeito da ligação entre o transtorno de ansiedade e o distúrbio da paralisia do sono na perspectiva da terapia cognitivo comportamental. A paralisia do sono é um distúrbio que acomete o sono e pode ser considerado relativamente comum, contudo, é pouco pesquisado e investigado. Segundo Denis et al. (2018), esse distúrbio é marcado pela experiência incomum de acordar durante a noite sem a capacidade de se mover. A grande maioria dos episódios está associado a medo extremo e, em uma minoria dos casos, pode levar a níveis clinicamente significativos de sofrimento.

A ocorrência deste distúrbio durante a noite pode vir a prejudicar a qualidade do sono, uma função biológica fundamental na consolidação da memória, na conservação e restauração da energia e do metabolismo energético cerebral. Portanto, devido a essas importantes funções, as perturbações do sono podem acarretar alterações significativas no funcionamento físico, ocupacional, cognitivo e social do indivíduo, além de comprometer substancialmente à qualidade de vida (MULLER E GUIMARÃES, 2017). De acordo com estudos científicos também existe uma relação estatisticamente significativa entre a qualidade de sono e os níveis de stress, ansiedade e depressão; indivíduos com sintomas de insônia reportam uma maior irritabilidade, desequilíbrios nas funções cognitivas, ansiedade, depressão, cansaço e um declínio na qualidade de vida (GALVÃO et al., 2017).

Os transtornos de ansiedade constituem o distúrbio de saúde mental mais comum vivenciado por jovens adultos. Os problemas relacionados ao sono são altamente prevalentes entre indivíduos ansiosos e abrange uma variedade de consequências, incluindo medos noturnos, insônia e recusa em dormir sozinho (WEINER et al., 2015).

O tema tem sua justificativa baseada na falta de informações a respeito da correlação entre a ansiedade e o distúrbio da paralisia do sono, tendo em vista que muitas pessoas que vivenciam o fenômeno da paralisia do sono se deparam com informações escassas e dados superficiais que não se aprofundam muito na raiz do problema e não mostram dados a respeito das possíveis causas geradoras deste distúrbio. Este é um assunto de extrema relevância, pois esse tipo de informação vem para ampliar o conhecimento sobre a temática abordada através de métodos científicos, trazendo 
clareza e melhor compreensão para a vida do indivíduo. Percebe-se então a partir disto, que é um estudo de extrema significância visando um âmbito social e individual do fenômeno.

A partir de tudo que foi ressaltado, o problema desta pesquisa foi, a ansiedade é uma potencial influenciadora do distúrbio da paralisia do sono? Já o objetivo deste trabalho se constituiu na investigação de como o transtorno da ansiedade pode estar ligado ao distúrbio da paralisia do sono trazendo a perspectiva da terapia cognitivo comportamental. No que se referem os objetivos específicos, estes foram: discutir o distúrbio da paralisia do sono e a ansiedade; compreender a relação entre a ansiedade e o distúrbio da paralisia do sono; identificar as consequências da ansiedade atrelada ao distúrbio da paralisia do sono no indivíduo e elaborar uma cartilha informativa sobre autocuidado ansiedade e paralisia do sono.

A respeito do método que foi utilizado para a condução desta pesquisa, este foi de caráter qualitativo. Visto que, a pesquisa de caráter qualitativo visa explicar os fenômenos sociais, possuindo uma natureza subjetiva. Esteve presente também nesta abordagem metodológica a pesquisa bibliográfica, que pode ser compreendida como o tipo de pesquisa que "abrange toda bibliografia já tornada pública em relação ao tema de estudo, desde publicações avulsas, boletins, jornais, revistas, livros, pesquisas, monografias, teses, material cartográfico etc.” (MARCONI; LAKATOS, 2003)

Já para a abordagem foi utilizado como base a terapia cognitivo comportamental, tendo em conta seus vastos estudos científicos a respeito do conhecimento e controle do transtorno da ansiedade.

\section{Definição e conceito de ansiedade e sua relação como o sono}

Em essência, a ansiedade significa a preservação da vida. É um mecanismo que faz parte da natureza enquanto defende e preserva o indivíduo. No entanto, passa a ser prejudicial e negativa quando funciona como um mecanismo neurótico, que visa defender a pessoa dos seus medos, como por exemplo, ser abandonada ou morrer; medos irracionais e que surgem a partir de percepções malformadas no inconsciente de um indivíduo (POSSATTO, 2006).

Ansiedade é um sentimento vago e desagradável de apreensão e medo, caracterizado por uma tensão ou desconforto que surge da antecipação da suposição de um perigo eminente, de algo desconhecido ou estranho. Esses sentimentos de ansiedade e medo passam a ser reconhecidos como patológicos quando são exagerados, desproporcionais em relação ao estímulo, ou qualitativamente diversos do que se observa normalmente naquela faixa etária e interferem com a qualidade de vida, o conforto emocional ou o desempenho diário do indivíduo. Tais reações exageradas ao estímulo 
ansiogênico se desenvolvem, mais comumente, em indivíduos com uma predisposição neurobiológica herdada (CASTILLO et al., 2000).

Um ponto importante a ser destacado também, é que a ansiedade não é um processo necessariamente ruim. Na medida em que é realizado, através dos pensamentos, o deslocamento do tempo presente para um tempo no futuro, o mecanismo da ansiedade se torna necessário para, por exemplo, planejar ações, situações, ou mesmo para se atingir metas (POSSATTO, 2006). Pode-se considerar então, a partir disto que foi trazido, que a ansiedade em sua medida correta é um mecanismo essencial para a manutenção do equilíbrio na vida de um indivíduo.

Ainda de acordo com Possatto (2006), pode-se dizer também que a ansiedade é um estado de alerta que se instala na mente de um indivíduo através dos pensamentos, alimentando o planejamento de nossas ações e buscando saídas, alternativas, e ensaiando ações de enfrentamento ou fuga. "É o preparo do corpo para fazer algo que o cérebro julga que precisa ser feito, mas que nem sempre o é" (POSSATTO, 2006 p.22).

No processo da ansiedade, o corpo responde ao que se pensa, e normalmente os pensamentos ansiosos negativos deslocam o indivíduo do aqui e agora para uma situação que, em geral, causa medo, preocupação ou apreensão. Na medida em que se pensa algo que amedronta, o cérebro recebe a mensagem de que precisa estar alerta para poder se defender; o corpo fica então preparado para enfrentar a situação, mas o que ocorre é que a situação não está acontecendo de fato, trata-se apenas de uma fantasia, assim, todo o preparo do corpo para atacar, reagir e se defender gerou uma energia que não foi utilizada concretamente. $O$ resultado de todo esse esforço é uma ansiedade negativa (POSSATTO, 2006).

Segundo o Manual Diagnóstico e Estatístico de Transtornos Mentais ( $5^{\underline{a}}$ edição, American Psychiatric Association, 2014, p.189),

Os transtornos de ansiedade incluem transtornos que compartilham características de medo e ansiedade excessivos e perturbações comportamentais relacionados. Medo é a resposta emocional a ameaça iminente real ou percebida, enquanto ansiedade é a antecipação de ameaça futura. Obviamente, esses dois estados se sobrepõem, mas também se diferenciam, com o medo sendo com mais frequência associado a períodos de excitabilidade autonômica aumentada, necessária para luta ou fuga, pensamentos de perigo imediato e comportamentos de fuga, e a ansiedade sendo mais frequentemente associada à tensão muscular e vigilância em preparação para perigo futuro e comportamentos de cautela ou esquiva. Às vezes, o nível de medo ou ansiedade é reduzido por comportamentos constantes de esquiva. Os ataques de pânico se destacam dentro dos transtornos de ansiedade como um tipo particular de resposta ao medo. Não estão limitados aos transtornos de ansiedade e também podem ser vistos em outros transtornos mentais. 
Então, resumidamente, os transtornos de ansiedade são transtornos que possuem como principais características medo e ansiedade em níveis excessivos, assim como perturbações comportamentais e possíveis ataques de pânico.

Os transtornos de ansiedade constituem o distúrbio de saúde mental mais comum vivenciado por jovens adultos. Os problemas relacionados ao sono são altamente prevalentes entre indivíduos ansiosos e abrangem uma variedade de consequências, incluindo medos noturnos, insônia e recusa em dormir sozinho. Distúrbios crônicos do sono estão associados a uma série de problemas comportamentais e físicos na juventude, o que pode levar a possíveis psicopatologias futuras ao longo da vida de um indivíduo (WEINER et al., 2015).

Weiner et al., (2015) traz também que, transtornos de ansiedade estão associados à deficiências funcionais e à redução da qualidade de vida ao longo do tempo, estão associados também a problemas relacionados ao sono. Existe uma relação recíproca entre as dificuldades do sono e a ansiedade, em que o sono perturbado aumenta a vulnerabilidade ao desenvolvimento da ansiedade, enquanto a ansiedade, por sua vez, interfere no sono.

A ansiedade patológica, em longo prazo, gera importantes consequências para a saúde, precipitando o surgimento de uma série de doenças. Essas doenças são desenvolvidas principalmente devido à ação persistente do aumento de tônus de adrenalina e de cortisol no corpo. O aumento prolongado de tônus adrenérgico pode levar inclusive a problemas físicos como a pressão alta, problemas cardíacos e disfunção nos vasos sanguíneos (ABUD, 2019).

Um episódio de ansiedade pode vir a causar episódios de medo intenso, pois produz no cérebro respostas semelhantes a certos perigos, chamada de Resposta de Luta ou Fuga (ABUD, 2019).

No âmbito dos estudos a respeito sobre ansiedade e sono, é importante salientar que a terapia cognitivo comportamental (TCC), se faz presente tentando identificações de tratamentos por meio de medidas comportamentais e mudanças de hábitos. Possatto (2006) traz que a única maneira de controlar nosso processo de ansiedade é reaprender posturas adequadas de vivência e pensamentos.

\subsection{O distúrbio da paralisia do sono e seus efeitos}

A paralisia do sono é um distúrbio que acomete o sono e pode ser considerado relativamente comum, contudo, é pouco pesquisado e investigado. Segundo Denis et al. (2018), esse distúrbio é marcado pela experiência incomum de acordar durante a noite sem a capacidade de se mover. A grande maioria dos episódios está associado a medo extremo e, em uma minoria dos casos, pode levar a níveis clinicamente significativos de sofrimento. 
De acordo com Ramos et al. (2019) a paralisia do sono ocorre quando a atonia do sono REM (movimento rápido dos olhos) continua ao despertar, sem outras características clínicas de narcolepsia. Episódios isolados de paralisia do sono são caracterizados por atonia muscular, com movimentos respiratórios e oculares preservados, no início ou final do sono, normalmente breves e que desaparecem espontaneamente ou mediante estímulo externo. Durante esta paralisia consciente, a maioria dos indivíduos têm sonhos vívidos, multissensoriais e, muitas vezes, com conotação negativa, tornando a paralisia do sono uma experiência muito desagradável.

Além das limitações das medidas usadas para avaliar a paralisia do sono, outro problema marcante é a falta de consistência com a terminologia. A paralisia do sono é um sintoma comum da narcolepsia, um distúrbio neurológico. A narcolepsia é caracterizada por sonolência diurna excessiva, cataplexia (perdas repentinas, breves e bilaterais de tônus muscular em resposta a emoções fortes como riso ou raiva) e padrões de sono noturnos perturbados. Portanto, o termo paralisia do sono é preferido quando a paralisia do sono está presente na ausência de um diagnóstico de narcolepsia (DENIS et al., 2018).

Ainda de acordo com Denis et al., (2018) uma revisão das taxas de prevalência da paralisia do sono ao longo da vida, na população geral, estimou a prevalência em aproximadamente $8 \%$, embora as estimativas de estudos individuais variem bastante de 2 a $60 \%$. A falta de uma medida mais padronizada para definir a paralisia do sono é provavelmente parte do motivo dessa variação extrema.

Outro patamar que precisa ser abordado quando se fala a respeito de um distúrbio do sono é o ato do dormir e o sono em geral. $O$ fato de dormir ser motivador e trazer novas energias sugere que o sono é uma necessidade vital. Estudos sobre a privação do sono realizados em humanos têm obtido evidências convincentes de que o ato de dormir bem é necessário para manter o pleno funcionamento normal do corpo (RIOS et al., 2008)

A qualidade do sono está diretamente associada ao estado de saúde e consequentemente à qualidade de vida, e pode ser associada como um indicador de saúde na comunidade. O mecanismo do sono é responsável por diversas funções no organismo do ser humano, e alterações nesse mecanismo, podem levar a consequências que influenciam no desequilíbrio emocional, que por sua vez, poderão originar perturbações depressivas, de ansiedade e stress (CERTO, 2016).

Diante disto, a ocorrência da paralisia do sono durante a noite pode vir a prejudicar a qualidade do sono, que por si só, é uma função biológica fundamental na consolidação da memória, na conservação e restauração da energia e do metabolismo energético cerebral. Portanto, devido a essas importantes funções, as perturbações do sono podem acarretar alterações significativas no 
funcionamento físico, ocupacional, cognitivo e social do indivíduo, além de comprometer substancialmente à qualidade de vida (MULLER E GUIMARÃES, 2017).

Existe uma ligação entre à qualidade do sono e a ocorrência da paralisia do sono. Indivíduos que classificam seu sono como muito bom ou bom, tem chances significativamente menores de sofrer paralisia do sono do que aquelas em que o sono é classificado como muito ruim ou ruim. A latência do sono e aumento da disfunção diurna está significativamente associada à paralisia do sono (DENIS et al., 2018).

A partir do que foi trazido nos últimos dois parágrafos, aponta que a má qualidade do sono noturno influencia para a ocorrência do distúrbio da paralisia do sono, assim como também o próprio distúrbio em si é influenciador da má qualidade do sono em geral.

O componente de interrupção do sono também é um fator significativo, fazendo com que se pressuponha que os sintomas de insônia são um preditor mais importante da paralisia do sono do que a qualidade geral do sono. Períodos prolongados de privação do sono por vezes levam a uma desorganização do ego, delírios e alucinações (RIOS et al., 2008).

Além dos aparentes danos psíquicos que a privação ou maus hábitos do sono aparentam causar, estudos realizando privação do sono em humanos, demonstraram importantes alterações metabólicas como hiperglicemia e quadros de resistência à insulina. Dados subsequentes sugerem que há um grande aumento no gasto energético. Possivelmente, estas alterações são influenciadas pelo significativo desbalanço hormonal que também ocorre sob esta condição; sendo o fígado um órgão importante para o metabolismo energético, hormonal e detoxificação, é possível que as alterações metabólicas observadas nos estudos de privação de sono envolvem o metabolismo hepático (RIOS et al., 2008).

Levando em consideração todos os dados expostos, a privação, má qualidade e maus hábitos do sono trazem danos mentais e físicos ao organismo de um indivíduo, tais enfermidades estas, que podem vir a afetar o mesmo, tanto em seu meio individual como também em seu meio coletivo. Denis et al. (2018, p.X) traz que "as causas da paralisia do sono são provavelmente multifatoriais. Portanto, é importante que os médicos e pesquisadores entendam os fatores que podem influenciar a frequência e a intensidade dos episódios”. A má higienização do sono é um desses fatores que podem vir a influenciar um distúrbio noturno.

Define-se higiene do sono como uma série de comportamentos, condições ambientais e outros fatores relacionados ao sono que podem ser prejudiciais durante a sua prática. Estudos demonstraram que a higiene inadequada do sono está intimamente associada a problemas para dormir, na qualidade e manutenção do sono. Indivíduos que desenvolvem um estilo de vida com 
bons hábitos noturnos, iniciam e mantém o sono em um processo natural. Esses hábitos e comportamentos têm efeitos positivos antes, durante e depois do horário de dormir. A higiene do sono é uma prática de coletânea de hábitos que além de trazer uma melhor qualidade de vida, por gerar uma qualidade de sono favorável, também é eficiente para eliminar a ansiedade gerada pelo medo antecipado de que o horário de dormir será estressante (SOCIEDADE BRASILEIRA DE PEDIATRIA, 2017).

O termo "higiene do sono" engloba modificações no ambiente do sono, práticas e rotinas favoráveis a um sono de melhor qualidade e de uma duração satisfatória. Além disso, inclui a prática de atividades no período de vigília que possam favorecer a chegada ao momento do sono, de maneira a favorecê-lo. Algumas dessas práticas são: possuir horários consistentes para deitar-se e despertar, tanto no sono noturno quanto no diurno e o estabelecimento de um local apropriado para se iniciar o sono (HALAL; NUNES, 2014).

Conforme o American academy of sleep medicine (2005), algumas práticas que costumam ser do cotidiano de muitos indivíduos, contribuem ativamente para a má higienização do sono, e assim a má qualidade do mesmo. Alguns exemplos dessas práticas são o consumo de álcool, cafeína, nicotina, refeições pesadas, consumo excessivo de aparelhos eletrônicos, exercícios físicos rígidos, horários irregulares para dormir ou a privação do sono, cansaço elevado e atividades psicologicamente estressantes desempenhadas antes do horário de dormir. Neste contexto, a Sociedade Brasileira de Pediatria (2017, p.I) traz que,

\begin{abstract}
A higiene do sono é utilizada em todas as idades para organizar o horário e os rituais de sono, ajudando também a evitar ou minimizar as parassonias, como sonambulismo, terror noturno, sonilóquio, pesadelos e algumas doenças do sono, tais como a Síndrome da Apneia Obstrutiva do Sono e a Síndrome das Pernas Inquietas. A Higiene do Sono associada a outras técnicas utilizadas na Terapia Cognitivo-Comportamental (restrição do sono e controle de estímulos), torna-se importante para o enfraquecimento de hábitos inadequados e para a instalação de rotinas que se associam ao dormir bem.
\end{abstract}

Portanto, a higiene adequada do sono é um guia para adequar comportamentos, e ajudar na prevenção de distúrbios do sono como a paralisia do sono.

\title{
3 Análise de dados
}

\section{I A relação da ansiedade com o distúrbio da paralisia do sono na perspectiva da terapia cognitivo comportamental.}

A Terapia Cognitivo Comportamental (TCC), traz em sua essência uma perspectiva de que o desenvolvimento de um estilo saudável de pensamentos e ações pode reduzir a angústia ou trazer uma maior sensação de bem-estar. Em suma, a abordagem se baseia em dois princípios centrais, que 
trazem que a cognição tem uma influência controladora sobre as emoções e comportamentos e o modo como se age ou se comporta pode afetar profundamente os padrões de pensamento $e$ as emoções (WRIGHT; BASCO; THASE, 2008).

De acordo com os autores Bahls e Navolar (2010), O funcionamento psicológico e a adaptação psicológica a determinados contextos estão na dependência de "estruturas de cognição com significado" ou simplesmente "esquemas". O termo "significado" é designado como o resultado da ação de interpretar uma realidade determinada e a forma como essa realidade se relaciona com o self. $\mathrm{O}$ ato de atribuir significado a algo ou a algum acontecimento é a adaptação a um contexto específico. Essa manobra adaptativa que ocorre a partir da atribuição de significados é uma função de "controle dos vários sistemas psicológicos" tais como o comportamental, o emocional, os sistemas de atenção e da memória. O que ocorre são influências entre os "sistemas cognitivos" e os demais sistemas psicológicos de forma a interagirem.

Ainda de acordo com os autores citados acima, uma "categoria de significado" apresenta implicações que são padrões específicos de emoção, atenção, memória e comportamento e tais efeitos podem ser denominados de "especificidade do conteúdo cognitivo". Pelo fato dos significados serem formados pelo indivíduo, não são componentes preexistentes da realidade, no entanto tais significados podem constituir-se como corretos ou incorretos em relação a um contexto ou objetivo específicos.

Os significados que podem ser classificados como incorretos são denominados como "disfuncionais" ou "mal adaptativos" no que se refere a ativação dos sistemas. Erros como distorções cognitivas e preconcepções, podem acontecer tanto em relação ao conteúdo cognitivo propriamente dito (significado) como em relação ao processo de elaboração de significado (processamento cognitivo) (BAHLS E NAVOLAR 2010).

Existe nos indivíduos uma predisposição a formação de construções cognitivas falhas e isto pode ser denominado como "vulnerabilidade cognitiva". Em decorrência da especificidade cognitiva uma vulnerabilidade cognitiva específica predispõe o indivíduo a uma síndrome específica. Os significados mal adaptativos, dos quais resultam a psicopatologia, são construídos em relação ao que é classificado como "Tríade Cognitiva", ou seja, em relação ao self, ao ambiente (experiência atual) e ao futuro (objetivo) (BAHLS; NAVOLAR 2010).

De acordo com a TCC, segundo Bahls e Navolar (2010), há dois tipos de significado: o "significado público" que pode não ter muitas implicações para o indivíduo (é o significado objetivo de um evento) e o "significado pessoal" ou privado. O significado pessoal é construído a partir de implicações e generalizações relacionadas a ocorrência de um evento. 
Existem três níveis de cognição: o nível pré-consciente, o nível consciente e o nível metacognitivo. O nível consciente desempenha o papel mais importante na melhora de um determinado indivíduo.

Os esquemas não são nunca adaptativos ou mal adaptativos sendo classificados assim com relação a um contexto específico. Assim os esquemas evoluem para facilitar a adaptação da pessoa ao ambiente no qual está inserida (BAHLS; NAVOLAR 2010).

Se faz importante trazer novamente um conceito que já foi abordado neste trabalho, de que a ansiedade é uma emoção. Freeman e Freeman (2014, P. x), trazem que "o conceito de "emoção" é controverso, porém há um acordo geral de que as emoções são fenômenos complexos, os quais afetam nossos pensamentos, nosso corpo e nosso comportamento."

A partir disso, retomando os dois princípios centrais sobre os quais a tcc se baseia, pode-se então dizer que, por ser uma emoção a ansiedade é diretamente influenciada a partir dos pensamentos e ações de um indivíduo, assim como também é diretamente influenciadora de padrões de pensamentos e comportamentos.

Um indivíduo que sofre de transtorno de ansiedade pode vir a atribuir significados mal adaptativos a uma determinada realidade. E sendo uma manobra adaptativa e uma função de controle de vários sistemas psicológicos, tais como o comportamental, o emocional e sistemas de atenção e memória, essa atribuição mal adaptativa a um significado pode gerar tensões que levam a estresses e até a maus comportamentos que podem vir a gerar episódios de insônia, pois como traz Weiner (2015), problemas relacionados ao sono são altamente prevalentes entre indivíduos ansiosos e abrangem uma variedade de consequências, incluindo medos noturnos, insônia e recusa em dormir sozinho, podendo assim contribuir para uma pré disposição a ocorrência de um episódio de paralisia do sono. De acordo com Rios (2008) a insônia é um dos preditores mais importantes para a ocorrência da paralisia do sono do que a qualidade geral do sono em geral, pois períodos prolongados de privação do sono por vezes levam a uma desorganização do ego, delírios e alucinações.

Existe uma relação recíproca entre as dificuldades do sono e a ansiedade, em que o sono perturbado aumenta a vulnerabilidade ao desenvolvimento da ansiedade, enquanto a ansiedade, por sua vez, interfere no sono. (WEINER et al., 2015).

De acordo com Bartholomeu et al. (2014), transtornos mentais, assim como a ansiedade, podem ser agravantes do desencadeamento de dependências do uso de substâncias. Segundo o American academy of sleep medicine (2005), algumas práticas rotineiras presentes no cotidiano de muitos indivíduos contribuem ativamente para a má higienização do sono, essa que por sua vez está intimamente associada a distúrbios noturnos, tal como a paralisia do sono. Alguns exemplos dessas 
práticas são o consumo de álcool, cafeína, nicotina, entre outras substâncias; horários irregulares para dormir ou a privação do sono, cansaço elevado e atividades psicologicamente estressantes desempenhada antes do horário de dormir.

De acordo com os conceitos da TCC abordados neste capítulo, o transtorno de ansiedade é o resultado da predisposição a formação de construções cognitivas falhas, podendo este feito ser denominado como "vulnerabilidade cognitiva". Bahls e Navolar (2010) trazem que uma vulnerabilidade cognitiva específica predispõe o indivíduo a síndromes e patologias. Sendo assim, poderia ser o distúrbio da paralisia do sono o resultado de uma formação de construções cognitivas falhas advindas do transtorno da ansiedade a partir de uma vulnerabilidade cognitiva.

\section{Resultados e discussões}

Após tudo que foi exposto neste trabalho, podemos concluir, a partir dos conceitos da TCC e a partir de pressupostos teóricos, que a ansiedade é uma influenciadora direta para a ocorrência do distúrbio da paralisia do sono, pois é geradora de situações que predispõem o aparecimento do mesmo. Contudo, o fato do distúrbio da paralisia do sono, de acordo com Certo (2016), afetar a qualidade do sono geral durante a noite, também torna o distúrbio um mecanismo influenciador para o desequilíbrio emocional e gerador de perturbações depressivas, de ansiedade e stress.

Seria correto afirmar então que assim como a ansiedade é uma potencial influenciadora para o surgimento do distúrbio da paralisia do sono, o próprio distúrbio em si também pode vir a ser um possível gerador ou potencial influenciador para o transtorno de ansiedade. Sendo assim, é possível concluir que um é potencializador e possível influenciador do outro, e de certa forma, ambos se complementam.

\section{Considerações finais}

Este trabalho teve como contribuição social o auxílio informativo acerca da paralisia do sono e também a cerca se sua ligação com o transtorno de ansiedade, visto que essa temática não é devidamente explorada, investigada e estudada. A contribuição científica também foi de grande valia uma vez que a escassez de assuntos a respeito da paralisia do sono é grande no meio dos estudos científicos a respeito do tema.

Buscou-se ao longo da realização desta pesquisa a compreensão sobre a temática abordada, trazendo ainda uma perspectiva da terapia cognitivo comportamental para contribuir com um enriquecimento a luz de uma abordagem pioneira em assuntos cognitivos comportamentais que está a todo momento contribuindo com estudos e explicações a respeito das subjetividades dos indivíduos. 
Por fim, pode-se considerar que ainda há muito a ser estudado a respeito do tema da paralisia do sono, e muitas contribuições ainda podem ser trazidas, tanto social quanto cientificamente em virtude que a possibilidade de temas neste ramo são amplas e ainda assim possuem poucas pesquisas e contribuições a respeito.

\section{Referências}

ABUD, Marco. Medo e Ansiedade. E-book, p. I-16. 2019. Disponível em: http://drmarcoabud.com.br/ebook-medo-e-ansiedade. Acesso em: is jun. 2020.

AMERICAN ACADEMY OF SLEEP MEDICINE. Internacional classification of sleep disorders: diagnostic and coding manual. 2nd ed. Wesrchester, 2005

BAHLS, Saint Clair; NAVOLAR, Ariana Bassetti Borba. TERAPIA COGNITIVOCOMPORTAMENTAIS: CONCEITOS E PRESSUPOSTOS TEÓRICOS. 2010 - Universidade Federal do Paraná e Unicenp - Paraná, 2010. Disponível em: https://psicoterapiaepsicologia.webnode.com.br/news/terapia-cognitivo-comportamentaisconceitos-e-pressupostos-teoricos/ Acesso em: 20 nov. 2020.

BARTHOLOMEU, Daniel et al. Avaliação da Ansiedade e outros aspectos emocionais de dependentes químicos em regime de internação. 2014. 34 v. - Academia Paulista de Psicologia, São Paulo, 2014. Disponível em: http://pepsic.bvsalud.org/scielo.php?script=sci_arttext\&pid=S 1415 7IIX2014000200005. Acesso em: 20 nov. 2020.

CERTO, Ana Catarina Trindade. Qualidade do sono e suas implicações ao nível da ansiedade, depressão e stress nos estudantes do ensino superior. 2016. I89 f. Tese (Doutorado) - Curso de Enfermagem Comunitária, Instituto Politécnico de Bragança, Bragança, Portugal, 2016. Disponível em: https://bibliotecadigital.ipb.pt/bitstream/10198/13051/r/TESE\%2oFINAL.pdf Acesso em: 31 out. 2020 .

CASTILLO, Ana Regina Gl et al., Transtornos de ansiedade. Brazilian Journal Of Psychiatry, São Paulo, v. 22, $\quad$ dez. $2000 . \quad$ Disponível em: https://www.scielo.br/scielo.php?script=sci_arttext\&pid=SI516-44462000ooo6oooo6\&lng=pt .Acesso em: 29 set. 2020.

DSM-V: Manual de Diagnóstico e Estatística das Perturbações Mentais ( $5^{\underline{a}}$ Ed.) American Psychiatric Association (2014).

DENIS, Dan. Relationships between sleep paralysis and sleep quality: current insights. US National Library of Medicine National Institutes of Health, 2 nov. 2018. Disponível em: https://www.ncbi.nlm.nih.gov/pmc/articles/PMC6220434/ . Acesso em: 19 jun. 2020.

FREEMAN, Daniel; FREEMAN, Jason. ANSIEDADE: o que é, os principais transtornos e como tratar. Porto Alegre: L\&pm, 2014. II8 p.

GALVÃO, Ana; PINHEIRO, Marco; GOMES, Maria José; ALA, Sílvia. Ansiedade, stress e depressão relacionados com perturbações do sono-vigília e consumo de álcool. Revista Portuguesa de Enfermagem de Saúde Mental, Portugal, ago. 2017. Disponível em: http://www.scielo.mec.pt/scielo.php?script=sci_arttext\&pid=Si647-21602017000200002 Acesso em: 08 maio 2020 . 
GIL, Antonio Carlos. Como elaborar projetos de pesquisa. São Paulo: Atlas, 2002.

HIGIENE DO SONO. Sociedade Brasileira de Pediatria (SBP) - Departamento científico de medicina do sono: n. I, nov. 2017. Disponível em: https://www.sbp.com.br/fileadmin/user_upload/r9807c-DocCient_-

_Higiene_do_Sono.pdf. Acesso em: 28 out. 2020.

HALAL, Camila S.e.; NUNES, Magda L.. Educação em higiene do sono na infância: quais abordagens são efetivas? Uma revisão sistemática da literatura. 2014. 90 v., Jornal de Pediatria, Rio de Janeiro/porto Alegre, 2014. Disponível em: https://www.scielo.br/scielo.php?pid=Soo2I$75572014000500449 \&$ script=sci_arttext\&tlng=pt. Acesso em: 28 out. 2020.

MARCONI, Marina de Andrade; LAKATOS, Eva Maria. Fundamentos de metodologia científica. 5. ed. São Paulo: Atlas, 2003. 310 p.

MULLER, Mônica Rocha; GUIMARÃES, Suely Sales. Impacto dos transtornos do sono sobre o funcionamento diário e a qualidade de vida - Curso de Psicologia, Departamento de Psicologia, Universidade de Brasília, Campus Universitário Darcy Ribeiro, Campinas, 2007. Disponível em:https://www.scielo.br/scielo.php?pid=so103-166×2007000400oII\&script=sci_arttext Acesso em: 08 maio 2020 .

POSSATTO, Lourdes. ANSIEDADE SOB CONTROLE. Lúmen, 2006.

RAMOS, Daniela Figueiredo et al. PARALISIA DO SONO RECORRENTE - MEDO DE DORMIR. Revista Paulista de Pediatria. São Paulo, 25 set. 2019. Disponível em: $\quad$ https://www.scielo.br/scielo.php?script=sci_arttext\&pid=Soro3$05822020000100603 \& \operatorname{lng}=p t \& n r m=$ iso \&thng $=$ pt .Acesso em: 13 jun. 2020.

RIOS, Alaíde Lílian Machado et al. Transtorno do sono, qualidade de vida e tratamento psicológico. UNIVERSIDADE VALE DO RIO DOCE/ FACULDADE DE CIÊNCIAS HUMANAS E SOCIAIS , Governador Valadares, p. I-53, 18 nov. 2008.

WEINER, Courtney L. et al. Anxiety Sensitivity and Sleep-Related Problems in Anxious Youth. US National Library of Medicine National Institutes of Health, I abr. 2015. Disponível em: https://www.ncbi.nlm.nih.gov/pmc/articles/PMC5340315/ Acesso em: I8 jun. 2020.

WRIGHT, Jesse H Wright; BASCO, Monica R; THASE, Michael E. APRENDENDO A TERAPIA COGNITIVO-COMPORTAMENTAL. São Paulo: Artmed, 2008. 219 p. 